\title{
Structural models for the design of novel antiviral agents against Greek Goat Encephalitis
}

The Greek Goat Encephalitis virus (GGE) belongs to the Flaviviridae family of the genus Flavivirus. The GGE virus constitutes an important pathogen of livestock that infects the goat's central nervous system. The viral enzymes of GGE, helicase and RNA-dependent RNA polymerase (RdRP), are ideal targets for inhibitor design, since those enzymes are crucial for the virus survival, proliferation and transmission. In an effort to understand the molecular structure underlying the functions of those viral enzymes, the three dimensional structures of GGE NS3 helicase and NS5 RdRP have been modelled. The models were constructed in silico using conventional homology modelling techniques and the known 3D crystal structures of solved proteins from closely related species as templates. The established structural models of the GGE NS3 helicase and NS5 RdRP have been evaluated for their viability using a repertoire of in silico tools. The goal of this study is to present the 3D conformations of the GGE viral enzymes as reliable structural models that could provide the platform for the design of novel anti-GGE agents. 
1 Structural models for the design of novel antiviral agents against Greek Goat Encephalitis

2 Louis Papageorgiou $^{1,2}$, Styliani Loukatou ${ }^{1}$, Vassiliki Lila Koumandou ${ }^{1}$, Wojciech Makałowski ${ }^{3}$,

3 Vasileios Megalooikonomou ${ }^{4}$, Dimitrios Vlachakis ${ }^{1, *}$, Sophia Kossida, ${ }^{1, *}$

$4 \quad{ }^{1}$ Computational Biology \& Medicine Group, Biomedical Research Foundation, Academy of Athens, Soranou

5 Efessiou 4, Athens 11527, Greece

$6 \quad{ }^{2}$ Department of Informatics and Telecommunications, National and Kapodistrian University of Athens, University

7 Campus, Athens, 15784, Greece

$8{ }^{3}$ Institute of Bioinformatics, University of Münster, Niels-Stensen-Straße 14 Münster 48149, Germany

$9{ }^{4}$ Computer Engineering and Informatics Department, University of Patras, Patras University Campus, 26504, Greece

$10 *$ Correspondence to:

11 Sophia Kossida \& Dimitrios Vlachakis

12 Bioinformatics \& Medical Informatics Team, Biomedical Research Foundation, Academy of

13 Athens, SoranouEfessiou 4, Athens 11527, Greece

14 Tel: + 302106597 199, Fax: +30 2106597545

15 E-mail: skossida@bioacademy.gr \& dvlachakis@,bioacademy.gr

16 Abstract 
17 The Greek Goat Encephalitis virus (GGE) belongs to the Flaviviridae family of the genus

18 Flavivirus. The GGE virus constitutes an important pathogen of livestock that infects the goat's central nervous system. The viral enzymes of GGE, helicase and RNA-dependent RNA polymerase (RdRP), are ideal targets for inhibitor design, since those enzymes are crucial for the virus' survival, proliferation and transmission. In an effort to understand the molecular structure underlying the functions of those viral enzymes, the three dimensional structures of GGE NS3 helicase and NS5 RdRP have been modelled. The models were constructed in silico using conventional homology modelling techniques and the known 3D crystal structures of solved proteins from closely related species as templates. The established structural models of the GGE NS3 helicase and NS5 RdRP have been evaluated for their viability using a repertoire of in silico tools. The goal of this study is to present the 3D conformations of the GGE viral enzymes as reliable structural models that could provide the platform for the design of novel anti-GGE agents.

\section{Introduction}

Greek Goat Encephalitis (GGE) is an endemic virus in Greece which infects the central nervous system of goats and leads to neurological disorders. As an arbovirus (arthropod-borne virus), GGE can be transmitted through ticks. In spite of the association of GGE with Tick-borne encephalitis (TBE) and Louping ill (LI) virus (Pavlidou et al. 2008) which are zoonotic viruses (transmitted from animals to humans), there are no verified zoonosis cases yet regarding the GGE virus (Vlachakis et al. 2013f). However, even though the GGE virus may not infect humans, the results of its epidemics are catastrophic, since national economies are closely related with livestock (Ataide Dias et al. 2008). GGE is a member of the Flaviviridae virus family (Papageorgiou et al. 2014; Pavlidou et al. 2008), a family with several species causing lethal vertebrate infections, through arthropod vector transmissions (Vlachakis \& Kossida 2013). Numerous important human and animal pathogens are classified in the Flaviviridae virus family. GGE belongs to the genus Flavivirus and specifically the group of Mammalian tick-borne encephalitis viruses (Vlachakis et al. 2013a). The GGE viral genome is a positive-sense RNA in a linear, single-stranded form, of about $10 \mathrm{~kb}$ length.

To date, no anti-GGE virus therapy is available, while many other fatal viruses of the Flaviviridae family also remain untreatable, so there is an urgent need for new antiviral drugs and vaccines (Vlachakis et al. 2012b). Based on recent genomic studies, Flavivirus RNA replication is associated with the non-structural proteins, such as NS3 helicase and NS5 RdRP, encoded by the C-terminal part of the viral polyprotein (Supplementary Figure 1) (Nulf \& Corey 2004). Viral RNA-dependent RNA polymerase is encoded by the NS5 domain and catalyzes the replication of

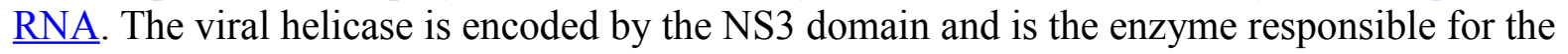
unwinding of the viral genetic material during replication. Therefore, the NS3 viral helicase and NS5 viral RdRP constitute ideal pharmacological targets, as they are key enzymes for the survival, propagation and eventual transmission of the virus (Vlachakis et al. 2012a). We propose that structural research and the establishment of a comprehensive in silico platform can aid the de novo design of novel inhibitors (Dalkas et al. 2013).

\section{Methods}

\section{Database sequence search}

59 NS3 helicase and NS5 RdRP sequence data from 87 species with fully sequenced genomes were 60 collected from the NIAID Virus Pathogen Database and Analysis Resource (ViPR) (Pickett et al.) 61 (http://www.viprbrc.org), and the NCBI RefSeq database, as described in (Vlachakis et al. 2013d)

62 In total, 7 Pestivirus, 8 Hepacivirus, 3 Pegivirus and 69 Flavivirus sequences were used, as 
63

64

65

66

67

68

69

70

71

72

73

74

75

76

77

78

79

80

81

82

83

84

85

86

87

88

89

90

91

92

93

94

95

96

97

98

99

100

101

102

103

104

105

106

shown in Supplementary Table 1. In cases where the NS5 annotation was not available in RefSeq, the start and end positions of the NS5 sequence within the whole genome polyprotein was inferred from CLUSTALW alignments with closely related annotated species; only the amino acid sequence corresponding to NS5, determined in this way, was used for the multiple sequence alignments and phylogenetic analysis (for details see Supplementary Table 1).

\section{Phylogenetic Analysis}

Alignments of the NS5 protein sequences (available upon request) were created using MUSCLE (Edgar 2004), and visualized using Jalview (Waterhouse et al. 2009). Only unambiguous homologous regions were retained for phylogenetic analysis; manual masking and trimming was performed in MacClade (Maddison \& Maddison 1989). The NS5 alignment was combined with data about NS3 helicase from our previous work (Vlachakis et al. 2013d) to create the tree based on both sequences. For each species, the NS3 helicase and NS5 RdRP sequences were concatenated after alignment, masking and trimming. This concatenated alignment was then tested with ProtTest (Abascal et al. 2005) to estimate the appropriate model of sequence evolution. Phylogenetic analysis was performed by Bayesian and Maximum likelihood methods as described in (Vlachakis et al. 2013d) with 100 bootstrap replicates. Trees were visualized using FigTree v1.4 (http://tree.bio.ed.ac.uk/software/figtree/).

\section{Molecular Modelling}

All calculations and visual constructions were performed on a six-core workstation running Windows 7 and using Molecular Operating Environment (MOE) version 2013.08 software package developed by Chemical Computing Group (Montreal, Canada).

\section{Identification of template structures and sequence alignment}

The amino acid sequence of the GGE genome polyprotein was retrieved from the conceptual translation of the virus genome sequence at the NCBI database (http://www.ncbi.nlm.nih.gov/) (genome sequence GenBank: DQ235153.1) (polyprotein sequence GenBank: ABB90677.1). The blastp algorithm (http://blast.ncbi.nlm.nih.gov/Blast.cgi) was used to identify homologous structures by searching in the Protein Data Bank (PDB) (Berman et al. 2002; Berman et al. 2013). The multiple sequence alignment was performed using MOE. Conserved residues in the multiple alignment were visualized with WEBLOGO (http://weblogo.berkeley. edu/logo.cgi).

\section{Homology Modelling}

The homology modelling of the Greek Goat Encephalitis viral RdRP and helicase were carried out using MOE. The selection of template crystal structures for homology modelling was based on the primary sequence identity and the crystal resolution. The MOE homology model method is separated into four main steps (Vlachakis et al. 2013e). First, a primary fragment geometry specification. Second the insertion and deletions task. The third step is the loop selection and the side-chain packing and the last step is the final model selection and refinement.

\section{Model optimization}

Energy minimisation was done in MOE initially using the Amber99 (Loukatou et al. 2014; Wang et al. 2000) force-field implemented into the same package, up to a root mean square deviation (RMSd) gradient of 0.0001 to remove the geometrical strain. The models were subsequently solvated with simple point charge (SPC) water using the truncated octahedron box extending to $7 \AA$ from the model, and molecular dynamics was performed at $300 \mathrm{~K}, 1 \mathrm{~atm}$ with 2 fsecond step size for a total of ten nanoseconds, using the NVT ensemble in a canonical environment (NVT stands for Number of atoms, Volume and Temperature that remain constant throughout the 
107 calculation). The results of the molecular dynamics simulation were collected into a database by

108 MOE for further analysis(Vlachakis et al. 2014).

109 Model evaluation

110 The produced models were initially evaluated within the MOE package by a residue packing 111 quality function, which depends on the number of buried non-polar side-chain groups and on 112 hydrogen bonding. Moreover, the suite PROCHECK (Laskowski et al. 1996; Papagelopoulos et 113 al. 2014) was employed to further evaluate the quality of the produced models. Finally, Verify3D 114 (Eisenberg et al. 1997; Luthy et al. 1992) was used to evaluate whether the models of NS3 115 helicase and NS5 RdRP domains are similar to known protein structures of this viral family 116 (Vlachakis et al. 2013b).

\section{Results}

\section{Phylogenetic analysis}

119 Alignment of the Flaviviridae NS5 protein sequences from a variety of Flaviviridae species 120 highlights novel important conserved functional domains (Supplementary Figure 2), as described 121 previously for NS3 helicase (Vlachakis et al. 2013d). Good conservation is evident throughout

122 the whole length of the sequence, particularly between species that belong to the same genus. The 123 annotated Pestivurs NS5 sequences ( 1215 aa) are significantly longer than the annotated NS5 124 sequences from Flavivirus ( $\sim 03$ aa) and Hepacivirus or Pegivirus ( 1039 aa). Pestivirus NS5 125 shows various internal insertions mostly throughout the N-terminal half of the protein. Regions 126 conserved across all three genera likely indicate important functional domains of the NS5 protein, 127 and the alignment highlights 13 residues which are absolutely conserved between all species. 128 Many of the conserved regions identified here have not been reported previously, and probably 129 deserve further study.

130 When data for NS3 helicase and NS5 RdRP is combined for phylogenetic reconstruction 131 across all 87 Flaviviridae species (Figure 1), the results are similar to our previous analysis based 132

133

134

135

136

137

138

139

140

141

142

143

144 on NS3 helicase (Vlachakis et al. 2013d). Clear separation of the different genera is seen. Within the Flavivirus, monophylletic groups for the tick-borne and insect-specific species are evident. TABV and the insect-specific Flavivirus appear basal to (closest to the origin of) the whole Flavivirus group. Mosquito-borne species diverge afterwards, followed by the tick-borne species, and species with no known vector (NKV). Most of the groupings in the tree are highly supported by posterior probability and bootstrap values by all three phylogenetic methods.

The tree highlights the phylogenetic distance between species for which structural information regarding NS3 helicase and NS5 RdRP is available. No structure is available from a tick-borne Flavivirus species for either NS3 helicase or NS5 RdRP, so our model proposed here for GGE fills a gap in structural information covering all Flaviviridae lineages. Given that Flaviviridae NS5 sequences for which structures are available come from phylogenetically disparate groups (see arrows in Figure 1) alignment of these structures allows fine-tuning of the structural model, and highlights conserved domains (Supplementary Figure 3).

\section{5}

146

\section{Structural models of the Greek Goat Encephalitis NS3 helicase and NS5 RdRP}

BLASTp against the PDB was used to identify the best available crystal structures as templates for homology modelling. Sequence alignments of the Greek goat encephalitis viral RdRP and viral helicase, to homologs from other Flaviviridae, identified several motifs that are important for biological functionality (Vlachakis et al. 2013c). A multiple sequence alignment (Supplementary Figure 3) was constructed including the Greek Goat Encephalitis NS5 viral 
151

152

153

154

155

156

157

158

159

160

161

162

163

164

165

166

167

168

169

170

171

172

173

174

175

176

177

178

179

180

181

182

183

184

185

186

187

188

189

190

191

192

193

194

195

196

197

198

RdRP, the crystal structure of Japanese Encephalitis NS5 RdRP with a conserved methyltransferase-polymerase interface (PDB code 4K6M) (Lu \& Gong 2013), the crystal structure of Dengue Virus NS5 RdRP bound to NITD-107 (PDB code 3VWS) (Noble et al. 2013), the crystal structure of Bovine Viral Diarrhea Virus NS5 RdRP (PDB code 1S48) (Choi et al. 2004) and the crystal structure of Hepatitis C NS5 RdRP with short RNA template strand (PDB code 1NB7) (O'Farrell et al. 2003).

The final choice of a template structure was not only based on the percent sequence identity, but also on the structure resolution. The Japanese Encephalitis virus RdRP and Yellow Fever helicase are suitable templates because both viruses belong to the same viral family with Greek Goat Encephalitis (Flaviviridae). The predicted secondary structure for the GGE virus RdRP was found to be similar to the actual structure of the JE virus RdRP; the same was true for the GGE viral helicase and the Yellow Fever viral helicase. The Japanese Encephalitis virus, Dengue virus, Hepatitis C virus and Greek Goat Encephalitis RdRPs all belong to the superfamily of RdRP and share eight common motifs within their domains. The Flaviviridae family helicases belong to the superfamily II of helicases and within their domains share seven common motifs. All RdRP and helicase invariant motifs have been conserved on the Greek Goat Encephalitis models (Lu \& Gong 2013)

The alignment between the RdRP sequence of the Greek Goat Encephalitis and the sequence of the Japanese Encephalitis virus RdRP (PDB 4K6M, resolution 2.6 A) (Lu \& Gong 2013) template revealed 58\% Identity and 73\% Similarity (Figure 2). The alignment for the viral helicase between the sequence of the Greek Goat Encephalitis and the sequence of the Yellow Fever virus (PDB 1YKS, resolution $1.8 \AA$ ) ( $\mathrm{Wu}$ et al. 2005) template revealed $50 \%$ Identity and $65 \%$ Similarity (Figure 3 ). These results allow for a conventional homology modelling approach to be considered. The two models of NS5 GGE RdRP and NS3 GGE helicase were first structurally superimposed and subsequently compared to their templates using the Swiss Pdb viewer (4.1.0) algorithm (Guex \& Peitsch 1997). The NS5 GGE RdRP model and the NS3 GGE helicase model exhibited an alpha-carbon RMSD of less than 0.78 and less than 1.35 angstroms, respectively. Furthermore, the models were evaluated in terms of their geometry with MOE and the Verify3D algorithm (Eisenberg et al. 1997). The compatibility of both the NS5 GGE RdRP model and the NS3 GGE helicase model, with their own amino acid sequence was assessed using the Verify3D algorithm. A structural class was delegated for each residue of every model based on its 3D environment and location (Vlachakis et al. 2013g). Each residue score value was calculated by the algorithm using a collection of reference structures. The scores for the GGE virus RdRP model ranged from +0.02 to +0.75 and for the GGE virus helicase ranged from -0.09 to +0.7 . According to these results, the two models can be characterized as having high quality; scores below +0.1 are indicative of serious problems in the models (Bujnicki et al. 2002). It was concluded that no poorly scored stretches of amino acids were included neither in structured nor conserved regions of the NS3 helicase and NS5 RdRP models (Supplementary Table 2 and Supplementary Figure 4).

\section{Description of the Greek Goat Encephalitis helicase model}

As predicted, from the sequence alignment of Greek Goat Encephalitis helicase (Figure 3) and its secondary structure prediction (data not shown), the constructed model presented the structural features of known Flaviviridae helicases. In the Greek Goat Encephalitis helicase model (Figure 4) the three distinct domains of helicases were structurally conserved, as well as the various motifs described by $\mathrm{Wu}$ (Wu et al. 2005). The seven sequence motifs of the GGE helicase occur in domain 1 and 2 (Figures 3, 4C and 4D) (Gorbalenya \& Koonin 1993). Helicase motif residues are involved in NTP binding and hydrolysis. Moreover, those motifs are involved in coupling the NTPase to RNA duplex unwinding by an unknown mechanism (von Hippel \& Delagoutte 2003).

Peer] reviewing PDF | (v2014:06:2270:1:1:NEW 15 Oct 2014) 
199

200

201

202

203

204

205

206

207

208

209

210

211

212

213

214

215

216

217

218

219

220

221

222

223

224

225

226

227

228

229

230

231

232

233

234

235

236

237

238

239

240

241

242

243

244

245

246

One of the most critical motifs in Flaviviridae helicases is the GxGKT/S motif 1 in domain 1, which in GGE is conserved in the same sequence position (loop) as in kinases. Motif 1 is also known as Walker A motif and its role involves binding of the $\beta$-phosphate of adenosine triphosphate (ATP) (Saraste et al. 1990). Based on site directed mutagenesis studies for Motif 1 , the mutant protein is inactive. Another crucial conserved motif for the GGE helicase is the DExH motif 2. The DExH motif also known as Walker B motif mediates the binding of the Mg2+-ATP substrate. The aspartate (Asp 170) was found to bind the Mg2+ and create the best conditions for nucleophilic attack through the optimum orientation of ATP (Ruff et al. 1991). Furthermore, motif 2 is expected to bind a bivalent cation associated with nucleotide $\gamma$-phosphate. Finally, another critical motif is the QRxGRxGR motif 5 in domain 2 which is right across the interdomain cleft from the DExH motif 2. Motif 5 is important to the Flaviviridae helicase function as it is involved in nucleic acid binding (Niedenzu et al. 2001).

The Greek Goat Encephalitis helicase model showed a very similar topology to its template, Yellow Fever helicase (Figures 4B, 4C and 4D). In general, the Flaviviridae viral helicases contain three domains, which are separated by two channels. Domains 1 and 3 have more interactions with each other than they have with domain 2. During the RNA chain unwinding, domain 2 is expected to undergo crucial movement rather than the other two domains. The channel between domains 1-2 and 3 accommodates the ssRNA during the viral RNA unwinding process. The helicase binds RNA in the specific arginine-rich site contained in the second domain (Luo et al. 2008). Specific contacts between domains 1 and 2 form the helicase motifs. In the NTPase catalytic site these contacts are expected to change, as the domains link together. Motifs 1a and 6 are in direct contact as the two domains are topologically closer. The linker connecting domains 1 and 2 is motif 3 . Finally motif 7 forms direct contacts with motifs 1,2 , and 3. The non-structural protein 3 (NS3) helicase has been reported to interact with other viral replication proteins including non-structural protein 5 (NS5) (Filomatori et al. 2006).

\section{Description of the Greek Goat Encephalitis RdRP model}

The Greek Goat Encephalitis RdRP secondary structure (data not shown) was predicted from its sequence alignment (Figure 2). The Greek Goat Encephalitis NS5 model produced of $\sim 900$ amino acids contained two main regions, the N-terminal S-adenosyl-L-methyltransferase dependent methyltransferase (MTase) region and the $\mathrm{C}$-terminal RdRP region (Figures 5A, 5B and 5D). The MTase region bears a 5' cap structure and is equally important as RdRP (Geiss et al. 2009). The MTase plays key roles in the capping process and is believed to catalyze the methylation steps and act as a guanylytrasferase to form the linkage (Ray et al. 2006). In the Greek Goat Encephalitis model a linker connects the MTase region and the RdRP region (Figures 5A, 5B, 5D, 6A1 and 6B). This connection is presented in the Japanese Encephalitis NS5 RdrRP, which is the template model of GGE as well as in other previous studies (Lu \& Gong 2013).

Flaviviridae non structural protein 5 (NS5) comprises eight conserved motifs (Lu \& Gong 2013) (Figure 7). The three domains of Greek Goat Encephalitis RdRP were found to be structurally conserved, as well as the various motifs and regions of Flaviviridae NS5(Figure 2). As with other viral Flaviviridae RdRP, Greek Goat Encephalitis RdRP is separated into the Nterminal extension, the main polymerase and the priming loop. The main polymerase adopts a shape analogous to a cupped right hand and contains the finger and thumb domains, which rise on the sides of the palm domain (Figure 6A1). The most conserved part of viral RdRP is the palm domain, which contains the motifs A, B, C and D (Lu \& Gong 2013). In motifs A and B there are two catalytic aspartic acid residues (D536 and D668) that are conserved in all single-subunit possessive polymerases (Gong \& Peersen 2010). The finger domain contains the motifs F and G. Despite the fact that these motifs have not been resolved, these RdRP structures exhibit high similarity to the NS5B that are represented in the other two genera of the Flaviviridae family 
247 (Gong \& Peersen 2010). The thumb domain of Greek Goat Encephalitis viral RdRP is relatively

248 divergent and contains only Motif E. The size of the thumb domain is larger than in other viral

249 Flaviviridae RdRPs because it carries additional elements (C-terminal extension) that facilitate

250 de novo initiation.

251 The Greek Goat encephalitis RdRP finger domain is separated into individual subdomains

252 according to Japanese Encephalitis (Lu \& Gong 2013) and Powassan Encephalitis RdRP

253 (Thompson \& Peersen 2004). In the finger domain four different subdomains have been marked,

254 the index finger, the middle finger, the ring finger and the pinky finger (Figures 6A2 and 6B).

255 The index finger comprises a nuclear localization signal that coincides with the recommended

256 binding site of the non-structural protein 3 (NS3) (Rawlinson et al. 2009). Moreover the thumb

257 domain interacts with the tip of the index finger and forms a unique encircled active site. The

258 second and the third strands of the fingers domain are in the middle finger area. No specific

259 function has been related with the middle finger area yet. The forth and the fifth strands of the

260 fingers domain are described as ring finger and this area includes the NTP binding of motif F.

261 Finally the pinky finger contains motif $\mathrm{G}$, which is relatively bulky and forms one side of the

262 dsRNA channel ( $\underline{\text { Gong \& Peersen 2010)}}$.

263 Conclusions

264 The 3D models of the Greek Goat Encephalitis viral enzymes were designed using homology

265 modelling. The X-ray crystal structure of the viral Yellow Fever helicase was employed as a

266 template for the Greek Goat Encephalitis viral helicase and the Japanese Encephalitis RNA

267 dependent RNA polymerase as a template for the Greek Goat Encephalitis RNA dependent RNA

268 polymerase. The models were evaluated and display high conservation of the functional domains

269 previously characterized in other Flaviviridae species. We therefore nominate our Greek Goat

270 Encephalitis enzymes models to be suitable for advanced, in silico de-novo drug design

271 experiments. In the future this drug discovery process may lead to the development of potential

272 inhibitor molecules.

273 Acknowledgements

274 We are indebted to the CamGrid computational resource on which the phylogenetic analyses

275 were performed. LP is grateful to prof. Maroulis Dimitrios for his continuous support throughout

276 his $\mathrm{PhD}$ research.

\section{Figures legend}

278 Figure 1: Phylogenetic reconstruction of Flaviviridae concatenated NS3 helicase and NS5

279 RdRP protein sequences. The tree shown is the best Bayesian topology. Numerical values at the 280 nodes of the tree $(\mathrm{x} / \mathrm{y} / \mathrm{z})$ indicate statistical support by MrBayes, PhyML and RAxML (posterior 281 probability, bootstrap and bootstrap, respectively). Values for highly supported nodes have been 282 replaced by symbols, as indicated. Nodes with at least 0.95 posterior probability and $80 \%$ 283 bootstrap support were considered robust, and nodes with at least 0.80 posterior probability and $28450 \%$ bootstrap support are indicated. The position of GGE is indicated by a red arrow. The 285 species for which 3D structure of NS5 RdRP are available are indicated with a blue arrow. The 286 yellow fever virus (YFV), for which the 3D structure of NS3helicase is available, is indicated 287 with a green arrow. Full details and accession numbers for all protein sequences used are given in 288 Supplementary Table 1. The tree confidently separates the Hepacivurs, Pestivirus, Pegivirus and

289 Flavivirus genera. Within the Flavivirus, TABV and insect-specific species appear basal, whereas

290 Tick-borne species and species with no known vector (NKV) are the most derived. 
291 Figure 2: Sequence alignment between the Greek Goat Encephalitis viral NS5 RdRP and 292 the corresponding sequence of the crystal structure of Japanese Encephalitis NS5 RdRP. All

293 eight major conserved motifs of Flaviviridae have been marked (Motifs A-G).

294 Figure 3: Sequence alignment between the Greek Goat Encephalitis viral NS3 helicase and 295 the corresponding sequence of the crystal structure of Yellow Fever NS3 helicase. All seven

296 major conserved motifs of Flaviviridae have been marked(M1, M1a, M2-M6).

297 Figure 4: Model of the Greek Goat Encephalitis virus helicase. A: Ribbon representation of 298 the produced Greek Goat Encephalitis virus helicase model. B: Ribbon representation of the 299

300

301

302

303

304

305

306

307

308

309

310

311

312

313

314

315

316

317 produced Greek Goat Encephalitis virus helicase model (colored Red) next to the corresponding Yellow Fever virus helicase (in green color). $\mathbf{C}$ and $\mathbf{D}$ : The conserved motifs of the Yellow Fever virus helicase (in green color) next to the corresponding motifs on the Greek Goat Encephalitis helicase model (colored in Red). The major motifs have been color-coded according to the conventions of Fig. 3, and are shown in CPK format (Usual space filling) along with the rest of the helicase motifs. E and F: Electrostatic surface potential for the Greek Goat Encephalitis helicase. Represented with red is the area of negative charge, with blue the area of positive charge and with white the un-charged region.

Figure 5: Model of the Greek Goat Encephalitis virus RdRP. A: Ribbon representation of the produced Greek Goat Encephalitis virus RdRP and N-terminal S-adenosyl-L-methyltransferase dependent methyltransferase (MTase) side view model (colored Yellow and green) next to the corresponding Japanese Encephalitis virus RdRP and MTase (in gold and blue color).The linker is shown in red. B: Ribbon representation of the produced Greek Goat Encephalitis virus RdRP (colored green) and MTase (colored yellow) side view complex model. The linker is shown in red. C and D: Electrostatic surface potential side view for the Greek Goat Encephalitis RdRP and MTase complex.

Figure 6: Global views of Greek Goat Encephalitis RdRP shown in different orientations. A1: Ribbon representation of the produced Greek Goat Encephalitis virus RdRP model, looking into the RdRP front channel (putative dsRNAchannel). The three main domains of RdRP are shown: thumb (colored violet), palm (gray) and finger (light blue), with the MTase region at the back (yellow).The linker is shown in red. A2: Ribbon representation of the produced Greek Goat Encephalitis virus RdRP model with more details about the finger domain subdomains, looking into the RdRP front channel (putative dsRNAchannel); B Side view of Ribbon representation of the produced Greek Goat Encephalitis virus RdRP model with more details about the finger domain subdomains. The linker between MTase and RdRP is shown in red.

324

325

326

327

328

329

330

331
Figure 7: Global views of Greek Goat Encephalitis RdRP shown in different orientations. The major motifs have been color-coded according to the conventions of Fig. 2, and are shown in CPK format (Usual space filling).

Supplementary Figure 1: Schematic diagram of the structural and non-structural proteins within the GGE genome polyprotein. The different complexes that are thought to arise in different cellular compartments during and following polyprotein processing are shown underneath.

Supplementary Figure 2: Alignment of Flaviviridae NS5 protein sequences. 
332

333

334

335

336

337

338

339

340

341

342

343

344

345

346

347

348

349

350

351

352

353

354

355

356

357

358

359

360

361

362

363

364

365

366

367

368

369

370

371

372

373

374

The alignment was generated using Muscle, and visualized with Jalview. Amino acids are colored blue based on percent identity in the alignment, the consensus weblogo is shown at the bottom.

Supplementary Figure 3: Multi-alignment of Flaviviridae NS5 protein sequences.

Supplementary Figure 4: GGE NS3 Helicase and NS5 RdRP models geometry assessment. The comparison of the two model's Ramachantran plot (A), their rotamer conformations (B) and corresponding Ramachandran plot of their templates (C) confirm that the two models are stereochemicaly viable and reliable.

Supplementary Table 1: Details and accession numbers of all sequences used for the alignments and phylogenetic analysis. The table also indicates species names abbreviations used in Figure 8 and 7.

\section{Supplementary Table 2: Model description and data validation.}

\section{References}

Abascal F, Zardoya R, and Posada D. 2005. ProtTest: selection of best-fit models of protein evolution. Bioinformatics 21:2104-2105.

Ataide Dias R, Mahon G, and Dore G. 2008. EU sheep and goat population in December 2007 and production forecasts for 2008. Eurostat, European Commission.

Berman HM, Battistuz T, Bhat TN, Bluhm WF, Bourne PE, Burkhardt K, Feng Z, Gilliland GL, lype L, Jain S, Fagan P, Marvin J, Padilla D, Ravichandran V, Schneider B, Thanki N, Weissig H, Westbrook JD, and Zardecki C. 2002. The Protein Data Bank. Acta Crystallogr D Biol Crystallogr 58:899-907.

Berman HM, Coimbatore Narayanan B, Di Costanzo L, Dutta S, Ghosh S, Hudson BP, Lawson CL, Peisach E, Prlic A, Rose PW, Shao C, Yang H, Young J, and Zardecki C. 2013. Trendspotting in the Protein Data Bank. FEBS Lett 587:1036-1045.

Bujnicki J, Rychlewski L, and Fischer D. 2002. Fold-recognition detects an error in the Protein Data Bank. Bioinformatics 18:1391-1395.

Choi KH, Groarke JM, Young DC, Kuhn RJ, Smith JL, Pevear DC, and Rossmann MG. 2004. The structure of the RNA-dependent RNA polymerase from bovine viral diarrhea virus establishes the role of GTP in de novo initiation. Proc Natl Acad Sci U S A 101:4425-4430.

Dalkas GA, Vlachakis D, Tsagkrasoulis D, Kastania A, and Kossida S. 2013. State-ofthe-art technology in modern computer-aided drug design. Brief Bioinform 14:745-752.

Edgar RC. 2004. MUSCLE: multiple sequence alignment with high accuracy and high throughput. Nucleic Acids Res 32:1792-1797.

Eisenberg D, Luthy R, and Bowie JU. 1997. VERIFY3D: assessment of protein models with three-dimensional profiles. Methods Enzymol 277:396-404.

Filomatori CV, Lodeiro MF, Alvarez DE, Samsa MM, Pietrasanta L, and Gamarnik AV. 2006. A 5' RNA element promotes dengue virus RNA synthesis on a circular genome. Genes Dev 20:2238-2249.

Geiss BJ, Thompson AA, Andrews AJ, Sons RL, Gari HH, Keenan SM, and Peersen OB. 2009. Analysis of flavivirus NS5 methyltransferase cap binding. J Mol Biol 385:1643-1654. 
375

376

377

378

379

380

381

382

383

384

385

386

387

388

389

390

391

392

393

394

395

396

397

398

399

400

401

402

403

404

405

406

407

408

409

410

411

412

413

414

415

416

417

418

419

420

421

422

423

424

425

426
Gong P, and Peersen OB. 2010. Structural basis for active site closure by the poliovirus RNA-dependent RNA polymerase. Proc Natl Acad Sci U S A 107:22505-22510.

Gorbalenya AE, and Koonin EV. 1993. Helicases: amino acid sequence comparisons and structure-function relationships. Curr Opin Struct Biol:412-429.

Guex N, and Peitsch MC. 1997. SWISS-MODEL and the Swiss-PdbViewer: an environment for comparative protein modeling. Electrophoresis 18:27142723.

Laskowski RA, Rullmannn JA, MacArthur MW, Kaptein R, and Thornton JM. 1996. AQUA and PROCHECK-NMR: programs for checking the quality of protein structures solved by NMR. J Biomol NMR 8:477-486.

Loukatou S, Papageorgiou L, Fakourelis P, Filtisi A, Polychronidou E, Bassis I, Megalooikonomou V, Makalowski W, Vlachakis D, and Kossida S. 2014. Molecular dynamics simulations through GPU video games technologies. Journal of Molecular Biochemistry 3:64-71.

Lu G, and Gong P. 2013. Crystal Structure of the full-length Japanese encephalitis virus NS5 reveals a conserved methyltransferase-polymerase interface. PLoS Pathog 9:e1003549.

Luo D, Xu T, Watson RP, Scherer-Becker D, Sampath A, Jahnke W, Yeong SS, Wang $\mathrm{CH}$, Lim SP, Strongin A, Vasudevan SG, and Lescar J. 2008. Insights into RNA unwinding and ATP hydrolysis by the flavivirus NS3 protein. EMBO J 27:32093219.

Luthy R, Bowie JU, and Eisenberg D. 1992. Assessment of protein models with threedimensional profiles. Nature 356:83-85.

Maddison WP, and Maddison DR. 1989. Interactive analysis of phylogeny and character evolution using the computer program MacClade. Folia Primatol (Basel) 53:190-202.

Niedenzu T, Roleke D, Bains G, Scherzinger E, and Saenger W. 2001. Crystal structure of the hexameric replicative helicase RepA of plasmid RSF1010. J Mol Biol 306:479-487.

Noble CG, Lim SP, Chen YL, Liew CW, Yap L, Lescar J, and Shi PY. 2013. Conformational flexibility of the Dengue virus RNA-dependent RNA polymerase revealed by a complex with an inhibitor. J Virol 87:5291-5295.

Nulf CJ, and Corey D. 2004. Intracellular inhibition of hepatitis C virus (HCV) internal ribosomal entry site (IRES)-dependent translation by peptide nucleic acids (PNAs) and locked nucleic acids (LNAs). Nucleic Acids Res 32:3792-3798.

O'Farrell D, Trowbridge R, Rowlands D, and Jager J. 2003. Substrate complexes of hepatitis C virus RNA polymerase (HC-J4): structural evidence for nucleotide import and de-novo initiation. J Mol Biol 326:1025-1035.

Papagelopoulos N, Vlachakis D, Filtisi A, Fakourelis P, Papageorgiou L, Megalooikonomou V, and Kossida S. 2014. State of the art GPGPU applications in bioinformatics. International Journal of Systems Biology and Biomedical Technologies 2:24-48.

Papageorgiou L, Vlachakis D, Koumandou VL, Papagelopoulos N, and Kossida S. 2014. Computer-Aided Drug Design and Biological Evaluation of Novel AntiGreek Goat Encephalitis Agents. International Journal of Systems Biology and Biomedical Technologies 2:1-16.

Pavlidou V, Gerou S, Diza E, Antoniadis A, and Papa A. 2008. Genetic study of the distribution of Greek goat encephalitis virus in Greece. Vector Borne Zoonotic Dis 8:351-354.

Pickett BE, Sadat EL, Zhang Y, Noronha JM, Squires RB, Hunt V, Liu M, Kumar S, Zaremba S, Gu Z, Zhou L, Larson CN, Dietrich J, Klem EB, and Scheuermann 
432

433

434

435

436

437

438

439

440

441

442

443

444

445

446

447

448

449

450

451

452

453

454

455

456

457

458

459

460

461

462

463

464

465

466

467

468

469

470

471

472

473

474

475

476

477
RH. 2012. ViPR: an open bioinformatics database and analysis resource for virology research. Nucleic Acids Res 40:D593-598.

Rawlinson SM, Pryor MJ, Wright PJ, and Jans DA. 2009. CRM1-mediated nuclear export of dengue virus RNA polymerase NS5 modulates interleukin-8 induction and virus production. J Biol Chem 284:15589-15597.

Ray D, Shah A, Tilgner M, Guo Y, Zhao Y, Dong H, Deas TS, Zhou Y, Li H, and Shi PY. 2006. West Nile virus 5 '-cap structure is formed by sequential guanine $\mathrm{N}-7$ and ribose 2'-O methylations by nonstructural protein 5. J Virol 80:8362-8370.

Ruff M, Krishnaswamy S, Boeglin M, Poterszman A, Mitschler A, Podjarny A, Rees B, Thierry JC, and Moras D. 1991. Class II aminoacyl transfer RNA synthetases: crystal structure of yeast aspartyl-tRNA synthetase complexed with tRNA(Asp). Science 252:1682-1689.

Saraste M, Sibbald PR, and Wittinghofer A. 1990. The P-loop--a common motif in ATPand GTP-binding proteins. Trends Biochem Sci 15:430-434.

Thompson AA, and Peersen OB. 2004. Structural basis for proteolysis-dependent activation of the poliovirus RNA-dependent RNA polymerase. EMBO J 23:34623471.

Vlachakis D, Argiro A, and Kossida S. 2013a. An update on virology and emerging viral epidemics. Journal Molecular Biochemistry 2:80-84.

Vlachakis D, Bencurova E, Papangelopoulos N, and Kossida S. 2014. Current State-ofthe-Art Molecular Dynamics Methods and Applications. Adv Protein Chem Struct Biol 94:269-313.

Vlachakis D, Karozou A, and Kossida S. 2013b. 3D Molecular Modelling Study of the H7N9 RNA-Dependent RNA Polymerase as an Emerging Pharmacological Target. Influenza Res Treat 2013:645348.

Vlachakis D, Kontopoulos DG, and Kossida S. 2013c. Space constrained homology modelling: the paradigm of the RNA-dependent RNA polymerase of dengue (type II) virus. Comput Math Methods Med 2013:108910.

Vlachakis D, and Kossida S. 2013. Molecular modeling and pharmacophore elucidation study of the Classical Swine Fever virus helicase as a promising pharmacological target. PeerJ 1:e85.

Vlachakis D, Koumandou VL, and Kossida S. 2013d. A holistic evolutionary and structural study of flaviviridae provides insights into the function and inhibition of HCV helicase. Peer] 1:e74.

Vlachakis D, Pavlopoulou A, Tsiliki G, Komiotis D, Stathopoulos C, Balatsos NA, and Kossida S. 2012a. An integrated in silico approach to design specific inhibitors targeting human poly(a)-specific ribonuclease. PLoS One 7:e51113.

Vlachakis D, Tsaniras SC, Feidakis C, and Kossida S. 2013e. Molecular modelling study of the 3D structure of the biglycan core protein, using homology modelling techniques. Journal Molecular Biochemistry 2:85-93.

Vlachakis D, Tsaniras SC, and Kossida S. 2012b. Current viral infections and epidemics of flaviviridae; lots of grief but also some hope. Journal Molecular Biochemistry 3.

Vlachakis D, Tsiliki G, and Kossida S. 2013f. 3D Molecular Modelling of the Helicase Enzyme of the Endemic, Zoonotic Greek Goat Encephalitis Virus. Springer 383:165-171.

Vlachakis D, Tsiliki G, Pavlopoulou A, Roubelakis MG, Tsaniras SC, and Kossida S. 2013g. Antiviral Stratagems Against HIV-1 Using RNA Interference (RNAi) Technology. Evol Bioinform Online 9:203-213.

von Hippel PH, and Delagoutte E. 2003. Macromolecular complexes that unwind nucleic acids. Bioessays 25:1168-1177. 
478 Wang J, Cieplak P, and Kollman P. 2000. How well does a restrained electrostatic 479 potential (resp) model perform in calculating conformational energies of $480 \quad$ organic and biological molecules. J Comp Chem 21:1049-1071.

481 Waterhouse AM, Procter JB, Martin DM, Clamp M, and Barton GJ. 2009. Jalview 482 Version 2--a multiple sequence alignment editor and analysis workbench. 483 Bioinformatics 25:1189-1191.

484 Wu J, Bera AK, Kuhn RJ, and Smith JL. 2005. Structure of the Flavivirus helicase: 485 486 implications for catalytic activity, protein interactions, and proteolytic processing. J Virol 79:10268-10277. 


\section{Figure 1 (on next page)}

Phylogenetic reconstruction of Flaviviridae concatenated NS3 helicaseand NS5 RdRP protein sequences.

[p] The tree shown is the best Bayesian topology.

Numerical values at the nodes of the tree $(x / y / z)$ indicate statistical support by MrBayes, PhyML and RAXML (posterior probability, bootstrap and bootstrap, respectively). Values for highly supported nodes have been replaced by symbols, as indicated. Nodes with at least 0.95 posterior probability and $80 \%$ bootstrap support were considered robust, and nodes with at least 0.80 posterior probability and $50 \%$ bootstrap support are indicated. The position of GGE is indicated by a red arrow. The species for which 3D structure of NS5 RdRP are available are indicated with a blue arrow. The yellow fever virus (YFV), for which the 3D structure of NS3helicase is available, is indicated with a green arrow. Full details and accession numbers for all protein sequences used are given in Supplementary Table 1. The tree confidently separates the Hepacivurs, Pestivirus, Pegivirus and

Flavivirus genera. Within the Flavivirus, TABV and insect-specific species appear basal, whereas Tick-borne species and species with no known vector (NKV) are the most derived. [p] 


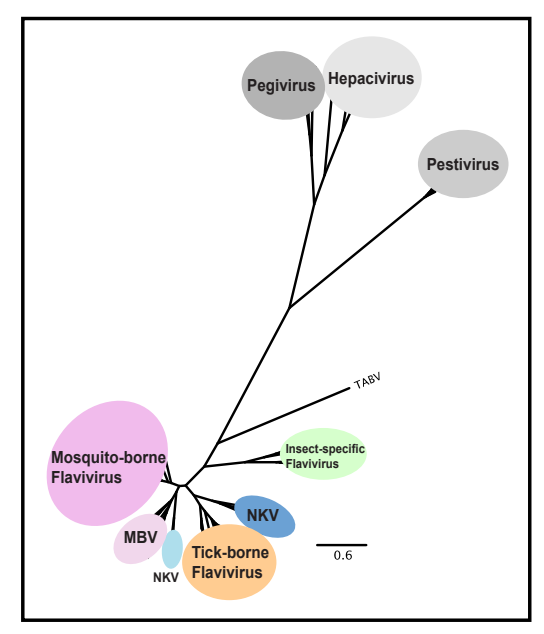

Peerd APriv

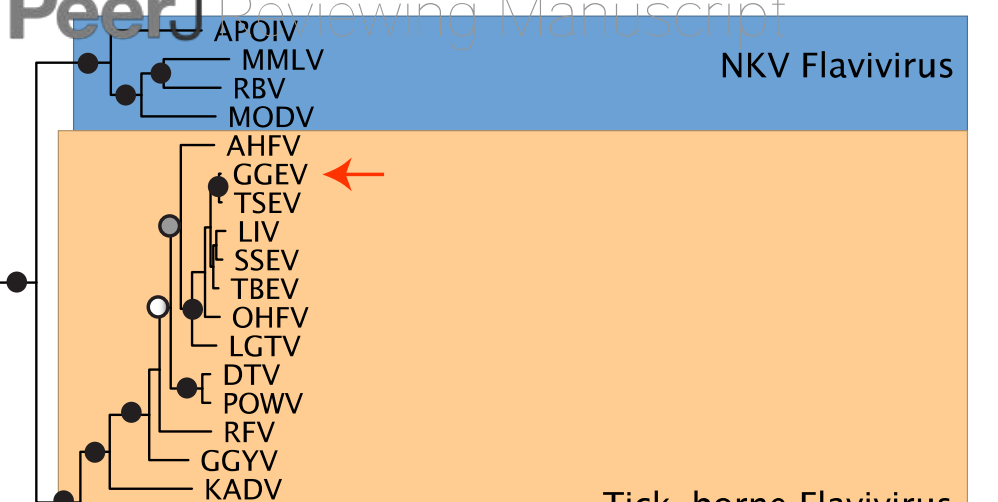

Tick-borne Flavivirus

T MEAV

TYUV

- SREV

- YNTV

BANV

NKV Flavivirus

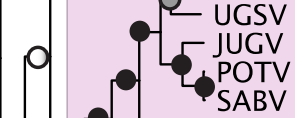

SABV
BOUV

EHV

드 WESSV

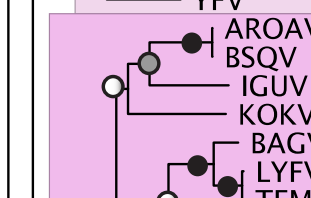

AROAV

BSQV

KOKV

TEMV

b) ILIHV

FVSP6

P
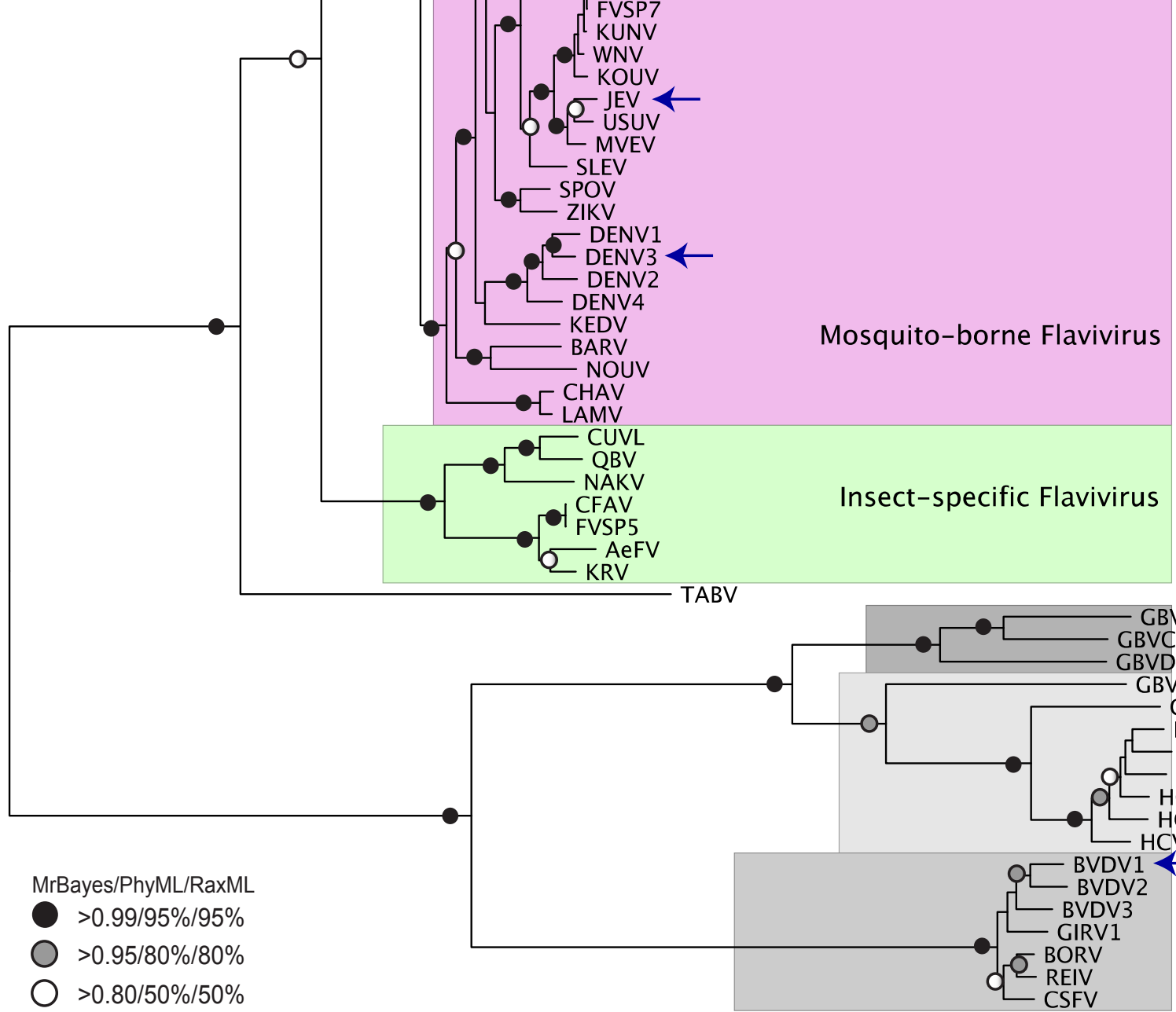

Pegivirus

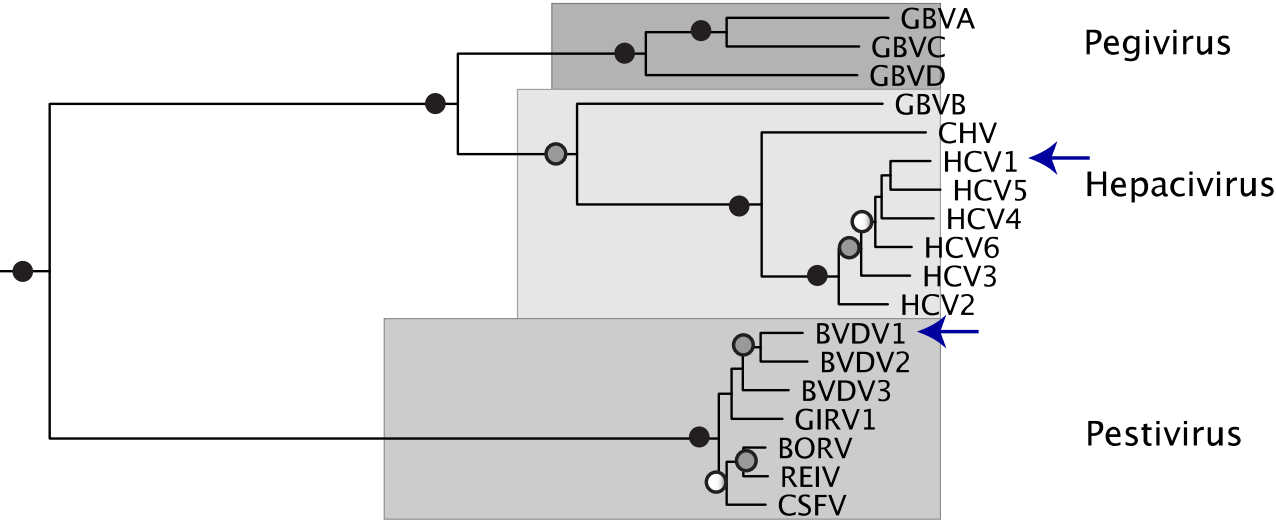


Figure 2 (on next page)

Sequence alignment between the GreekGoat Encephalitis viral NS5 RdRP and the corresponding sequence of the crystalstructure of Japanese Encephalitis NS5 RdRP.

All eight major conserved motifs of Flaviviridae have been marked (Motifs A-G). 


\section{PeerJ Reviewing Manuscript}

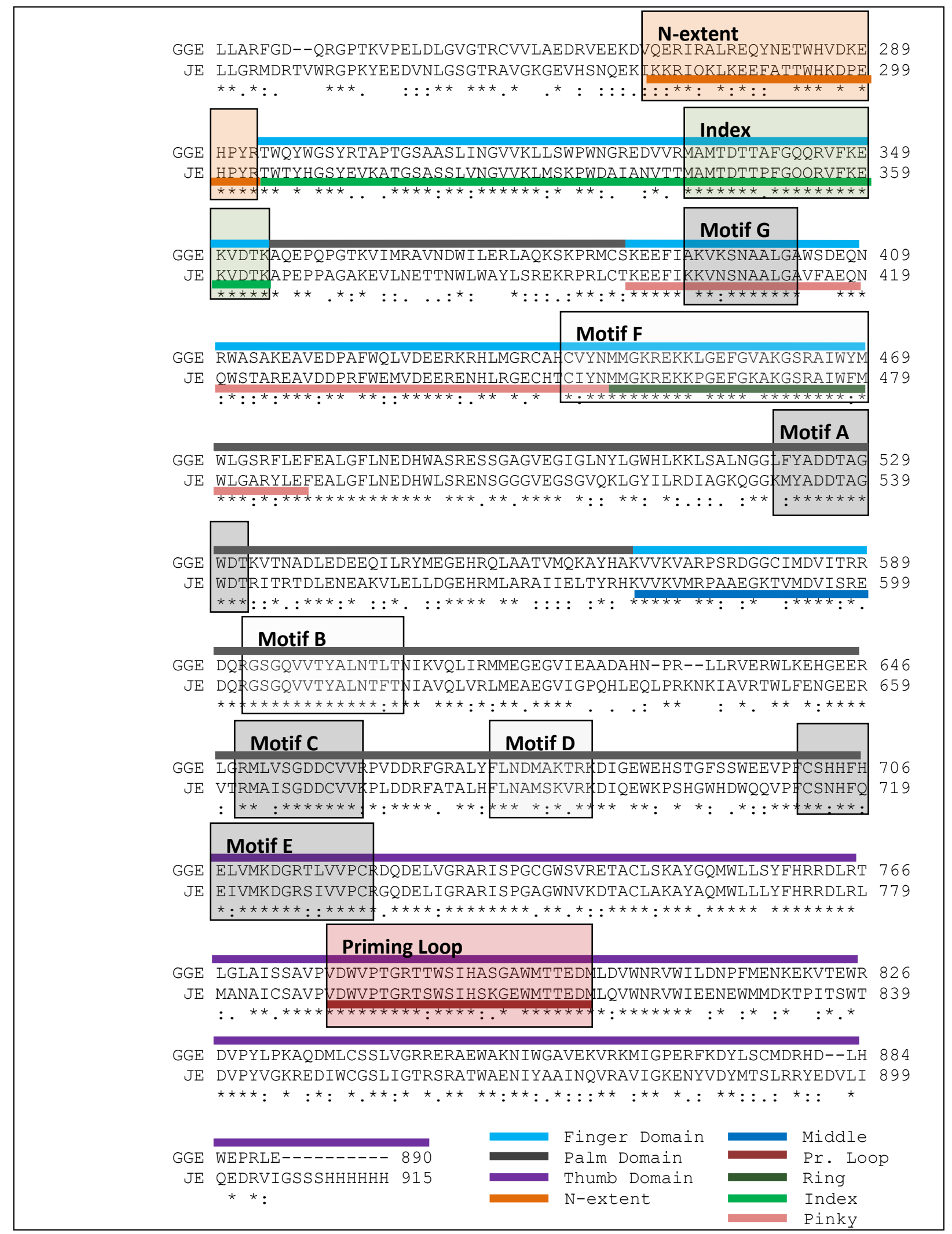




\section{Figure 3 (on next page)}

Sequence alignment between the GreekGoat Encephalitis viral NS3 helicase and the corresponding sequence of thecrystal structure of Yellow Fever NS3 helicase.

All seven major conserved motifs of Flaviviridae have been marked (M1, M1a, M2-M6). 


\section{PeerJ Reviewing Manuscript}

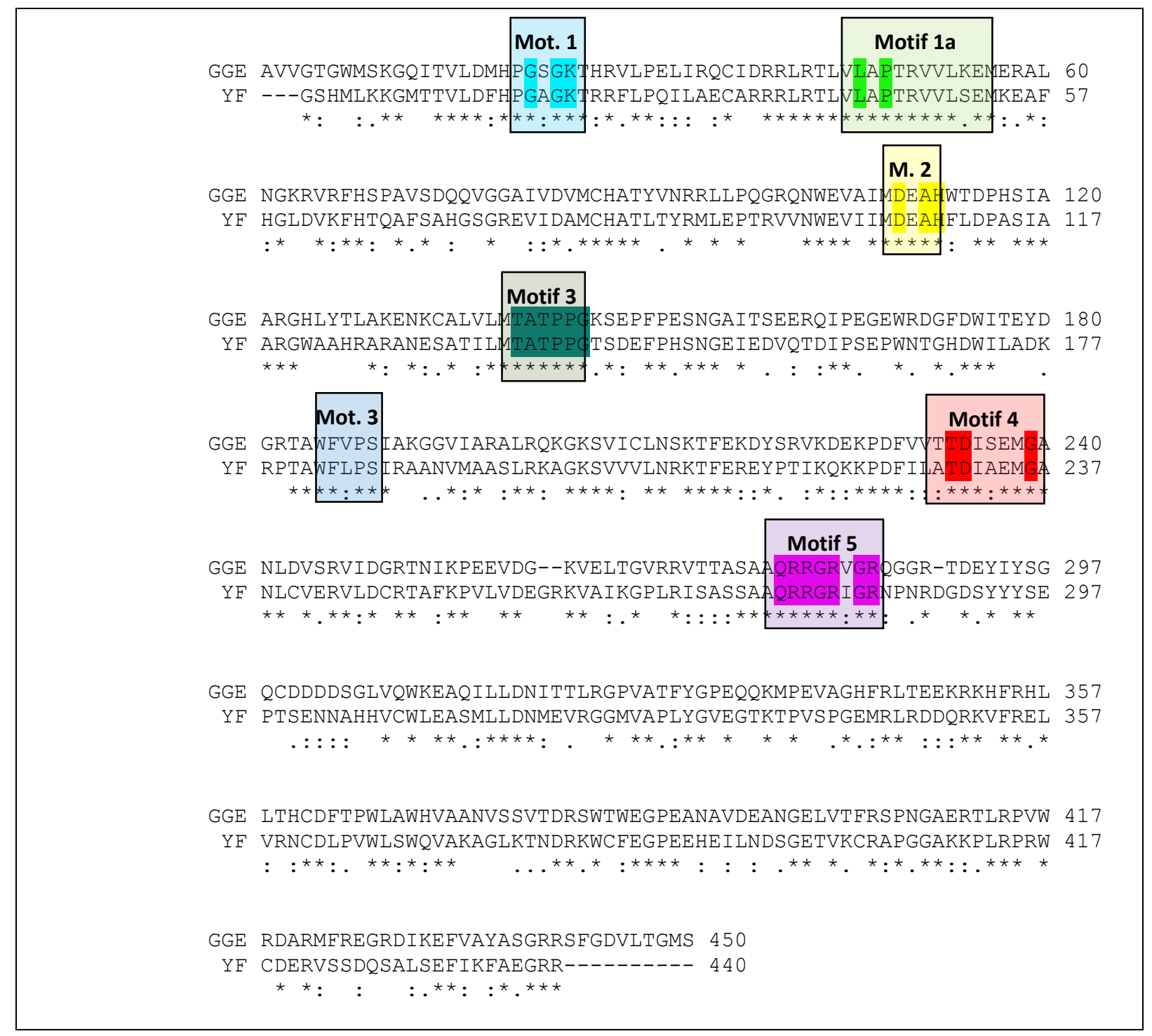




\section{Figure 4}

NS3 Model of the Greek Goat Encephalitisvirus helicase.

[p] A : Ribbon representation of the produced Greek Goat Encephalitis

virus helicase model. B: Ribbon representation of the produced Greek

Goat Encephalitis virus helicase model (colored Red) next to the corresponding

Yellow Fever virus helicase (in green color). C and D: The

conserved motifs of the Yellow Fever virus helicase (in green color) next to

the corresponding motifs on the Greek Goat Encephalitis helicase model (colored

in Red). The major motifs have been color-coded according to the conventions of

Fig. 3, and are shown in CPK format (Usual space filling) along with the rest

of the helicase motifs. $\mathbf{E}$ and $\mathbf{F}$ : Electrostatic surface potential

for the Greek Goat Encephalitis helicase. Represented with red is the area of negative charge, with blue the area of positive charge and with white the un-charged region. [p] 

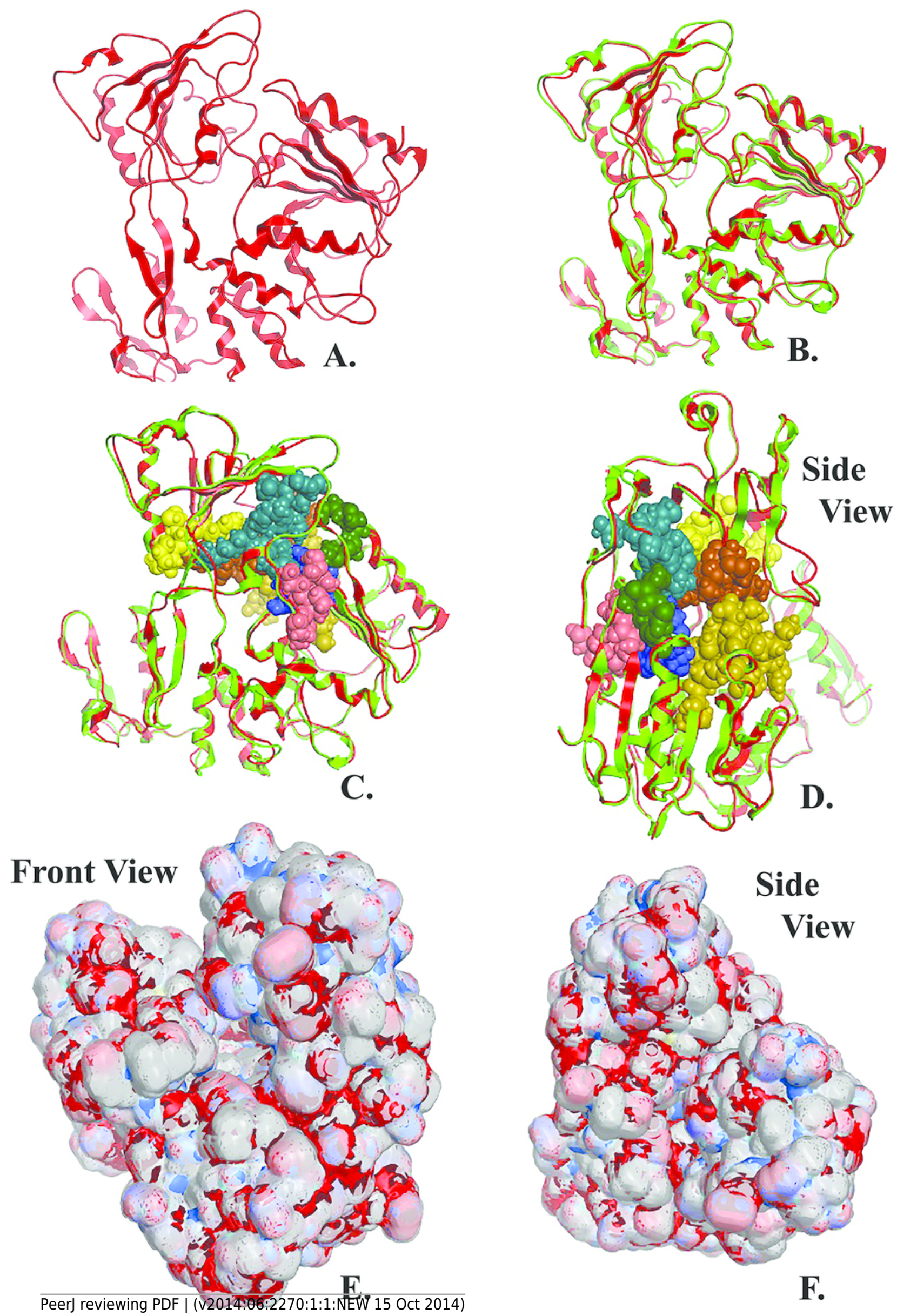


\section{Figure 5}

NS5 Model of the Greek GoatEncephalitis virus RdRP.

[p] A : Ribbon representation of the produced Greek Goat Encephalitis

virus RdRP and N-terminal S-adenosyl-L-methyltransferase dependent

methyltransferase (MTase) side view model (colored Yellow and green) next to the corresponding Japanese Encephalitis virus RdRP and MTase (in gold and blue color).The linker is shown in red. B: Ribbon representation of the produced Greek Goat Encephalitis virus RdRP (colored green) and MTase (colored yellow) side view complex model. The linker is shown in red. $\mathbf{C}$ and $\mathbf{D}$ :

Electrostatic surface potential side view for the Greek Goat Encephalitis RdRP and MTase complex. [p] 

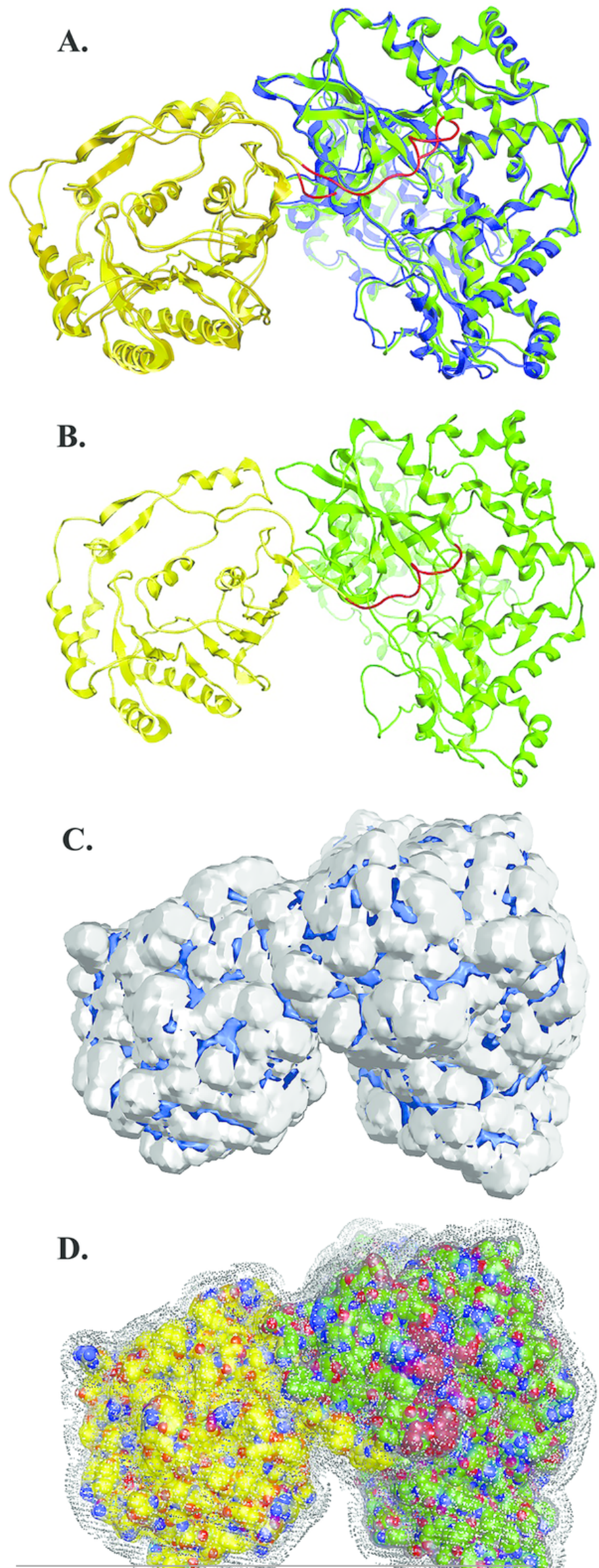


\section{Figure 6}

Global views of Greek Goat EncephalitisNS5 RdRP shown in different orientations.

[p] A1: Ribbon representation of the produced Greek Goat Encephalitis virus RdRP model, looking into the RdRP front channel (putative dsRNAchannel). The three main domains of RdRP are shown: thumb (colored violet), palm (gray) and finger (light blue), with the MTase region at the back (yellow).The linker is shown in red. A2: Ribbon representation of the produced Greek Goat Encephalitis virus RdRP model with more details about the finger domain subdomains, looking into the RdRP front channel (putative dsRNAchannel); B Side view of Ribbon representation of the produced Greek Goat Encephalitis virus RdRP model with more details about the finger domain subdomains. The linker between MTase and RdRP is shown in red. [p] 

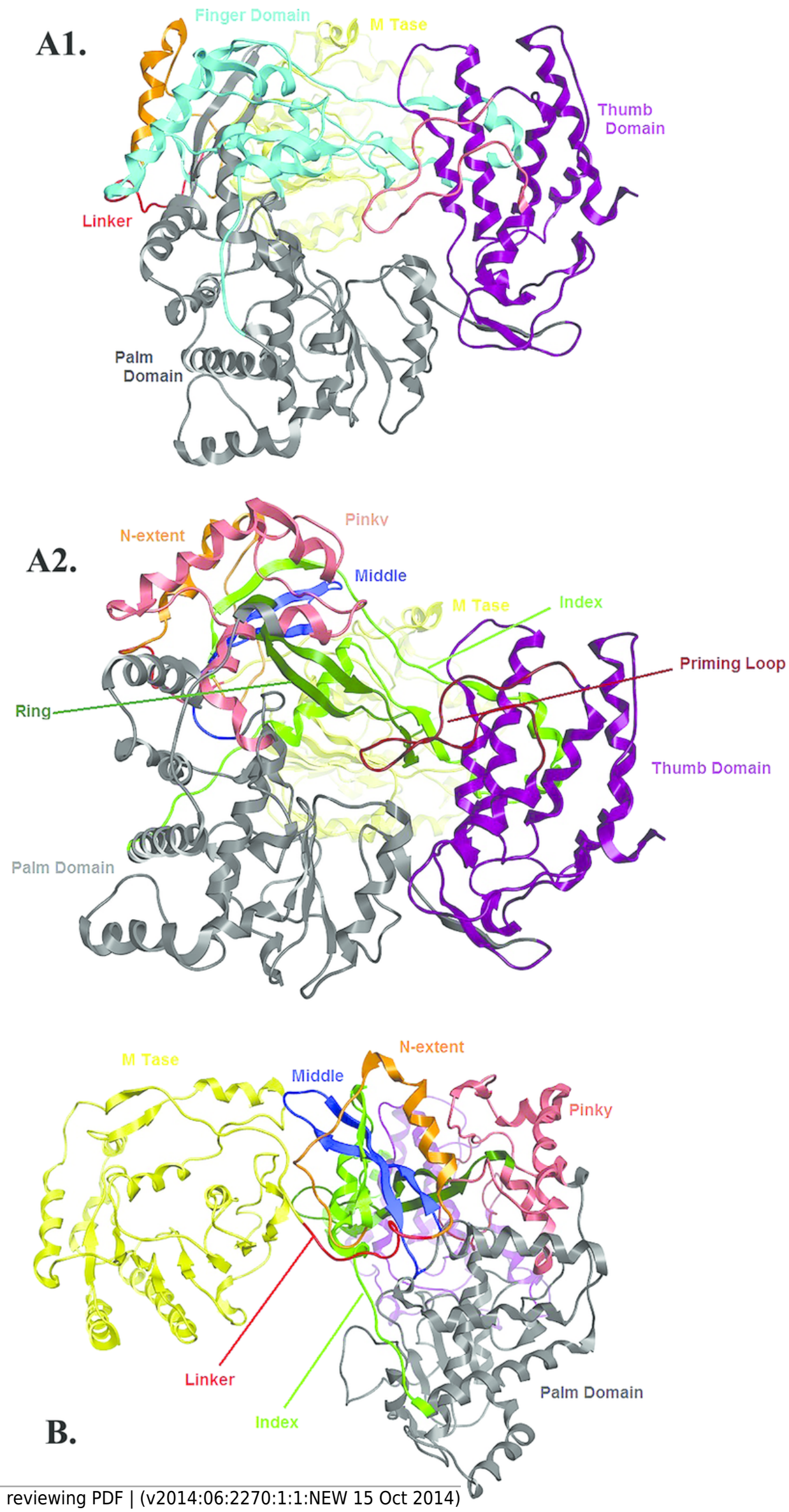


\section{Figure 7}

Global views of Greek Goat EncephalitisNS5 RdRP shown in different orientations.

[p] The major motifs have been color-coded

according to the conventions of Fig. 2, and are shown in CPK format (Usual

space filling). [p] 


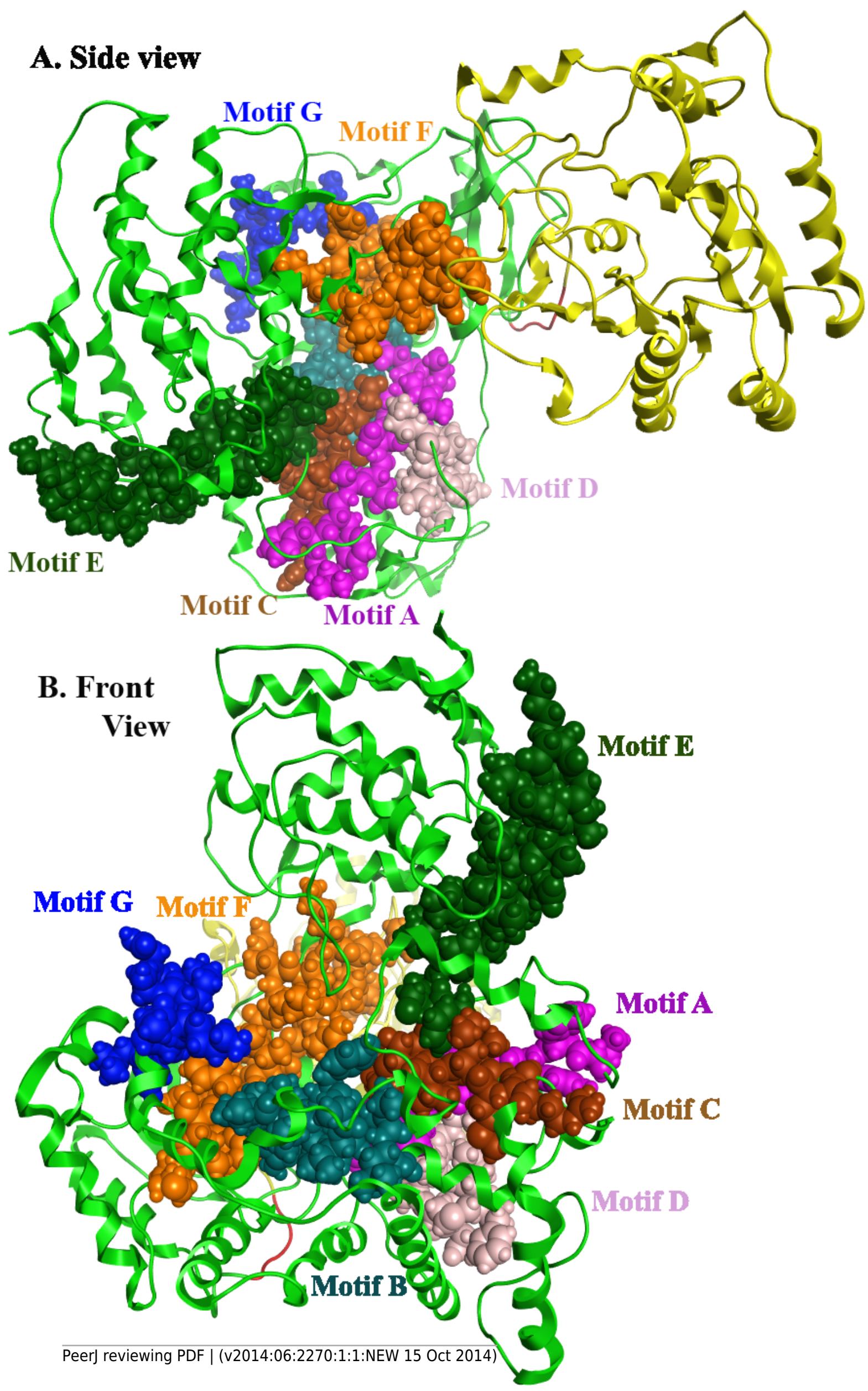

